\title{
Cavitation Bubble Dynamics during Laser Wet Etching of Transparent Sapphire Substrates by $1064 \mathrm{~nm}$ Laser Irradiation
}

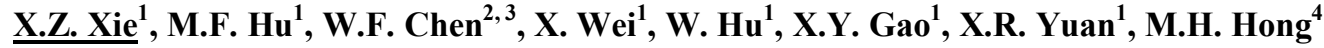 \\ 1- School of Electromechanical Engineering, Guangdong University of Technology, Guangzhou, Guangdong, China 510006 \\ 2 - College of Mechanical Engineering, Nanjing University of Aeronautics and Astronautics, Nanjing Jiangsu, China 210016 \\ 3 - Jiangsu Key Laboratory of Precision and Micro-manufacturing Technology, Nanjing Jiangsu, China 210016 \\ 4- Department of Electrical and Computer Engineering, National University of Singapore, Singapore 117576
}

E-mail:xiaozhuxie@gdut.edu.cn

\begin{abstract}
Laser induced backside wet etching (LIBWE) has shown to be a promising method to process transparent sapphire substrates. However, the etching rates of the conventional LIBWE are not high enough. Our recent experiments indicated that decreasing the gap between the sapphire and the confined plate to a certain value can enhance the etching rates and improve the surface quality. In this paper, we conduct the numerical simulation and the high speed photography imaging to explore the mechanism of the enhanced etching rate processed by $1064 \mathrm{~nm}$ pulsed laser in $\mathrm{CuSO}_{4}$ solution. The volume-of-fluid method (VOF) is used to compute the growth and collapse of the laser induced cavitation bubbles being attached to the rear sapphire surface and the flow field distribution in the gap between the sapphire and the confined plate. The cavitation bubble's dynamics at different gap heights are observed by high speed photography technique. Both the numerical and experimental data indicate that with the decreasing gap height, the bubble lifetime increases and the flow field distribution is favorable to enhance the liquid generating photochemical deposition on the rear sapphire surface. Furthermore, the absorption to laser increases, which leads to the increasing of the etching rate. The faster liquid convection at smaller gap height is conducive to heat dissipation around the processed zone, which is good to improve the surface quality.

DOI: $10.2961 / \mathrm{j} \mathrm{lmn} .2013 .03 .0012$
\end{abstract}

Key words: Laser induced backside wet etching, cavitation bubble dynamics, numerical simulation, high speed photograph imaging

\section{Introduction}

Sapphire has been used for a broad of applications, for example, precision instruments, military elements, optical window components of medical instruments, LED substrates and the micro-structures, because the transparent material has many advantages including good mechanical properties, good optical \& chemical properties, and high electrical insulation ${ }^{[1,2]}$. Meanwhile, these properties make it difficult to fabricate micron-sized structures on sapphire surfaces. The "classical" approach is the diamond blade cutting and chemical etching method. However, the diamond blade cutting is easily to create cracks and fragments. Chemical etching is time consuming and the use of strong acid or alkali will pollute the environment.

Laser-induced backside wet etching (LIBWE) has become more and more popular recently. The working liquid can cool the material surface and take away the debris during the processing of materials. However, the etching rate of the conventional LIBWE is not high enough. In the typical LIBWE process, a laser beam passes through the substrate without being absorbed at the upper surface and impinges on the absorbing liquid that is in contact with backside of material ${ }^{[3]}$. A high absorption coefficient organic $^{[4]}$ or inorganic metal salt solutions ${ }^{[5]}$ are commonly selected as working liquids. When metal salt solution is used, the absorbing liquid is thermally degraded, leaving a thin metal or metal oxide layer on the rear substrate. The metal or metal oxide layer enhances the absorption to the successive incident laser. K. Zimmer ${ }^{[6]}$ investigated the influence of the laser spot size and the applied pulse number to the etch rate for fused silica, and two different absorbing liquids $(0.5 \mathrm{~mol} / 1$ pyrene/toluene, $0.5 \mathrm{~mol} / \mathrm{l}$ pyrene $/ \mathrm{C}_{2} \mathrm{Cl}_{4}$ ) were selected as working liquids. His findings indicated that the bubble has an influence on the etch rate. Gonzalez-Avila ${ }^{[7]}$ studied the cavitation bubble dynamics at a variable gap height. Our recent experiments indicated that decreasing the gap between the sapphire and the confined plate to a certain value can enhance the etching rates and improve the surface quality. Consequently, it is evident that the influence on the laser-induced cavitation bubbles dynamics by the variable gap height during the LIBWE needs to be elucidated clearly.

Studying the bubbles dynamics usually include experimental method or numerical simulation. Recently detecting and measuring techniques for experimental studying the bubble dynamics have been developed in two primary aspects, including the time-varying bubble shape (e.g. high-speedphotography, interferometry, fiber-optical sensor based on optical beam deflection), and the timevarying pressure field around the oscillating bubble (e.g. hydrophone, piezoelectricity sensor, force sensor based on a fiber-optical sensor). High-speed photography method ${ }^{[8]}$ is able to record each 
transient cavitation bubble and liquid flow image, and the whole change process of bubbles form and the bubble pulsation cycle can be gained through analyzing the pictures and the number of frames.

The main numerical simulation techniques are listed as follows: boundary element method (BEM) ${ }^{[9]}$, level set method (LSM) ${ }^{[10]}$, volume-of-fluid method (VOF) ${ }^{[11]}$, lattice-Boltzmann (LBM) ${ }^{[12]}$ and coupled level set /volume-of-fluid (CLSVOF) ${ }^{[13]}$.

In this paper, we conduct the numerical simulation and the high-speed-photography technology to study the cavitation bubble dynamics during laser wet etching of transparent sapphire substrates by $1064 \mathrm{~nm}$ laser irradiation in $\mathrm{CuSO}_{4}$ solution. The varied gap height between the sapphire and the confined plate is of $0.43,1.25,2.5,3.75$ and $10 \mathrm{~mm}$, respectively. The volume-of-fluid method (VOF) is used to compute the growth and collapse of the laser induced cavitation bubbles being attached to the rear sapphire surface and the flow field distribution. The hydrodynamics in $\mathrm{CuSO}_{4}$ solution are visualized using high-speed-photography method. Finally, a validation experiment is conduced.

\section{Experiment}

\subsection{Experimental setup}

Figure 1 shows the experimental setup for the observation of cavitation bubble dynamics during laser wet etching of transparent sapphire substrates by high speed photography technique. The experimental setup consists of a laser system, imaging and illumination devices. A Q-switched Nd: YAG laser (wavelength $1064 \mathrm{~nm}$, a pulse duration $100 \mathrm{~ns}$ ) is used. Laser beam is reflected by a galvanometer, which can ensure the focal plane of the laser beam on the target surface by the rotating of the attached X \& Y mirrors. Laser beam then passes through the sapphire sample, focuses on the solidliquid interface.

Pulse frequency used in the experiment is fixed at $2 \mathrm{kHz}$, the single pulse energy ranges from 0 to 10 $\mathrm{mJ} /$ pulse at this time. The laser focal spot diameter is of $100 \mu \mathrm{m}$.

A piece of glass as a confined plate is placed below the sapphire substrate. The gap height between the sapphire and the confined plate is defined as $\mathrm{H}$, which is adjustable. The laser induced cavitation bubble diameter along $\mathrm{Y}$ direction is regarded as $\mathrm{D}_{\mathrm{y}}$. Besides, the gap is filled with $\mathrm{CuSO}_{4}$ solution without any additives, whose concentration is $20 \%$.

\subsection{Characterization}

A sapphire plate (Chongqing Shuzhou technology Co., Ltd) with a thickness of about $0.43 \mathrm{~mm}$ is used as a sample. The movements of the cavitation bubble are recorded with a high-speed camera (HiSpec5, Fastec Imaging Corporation)

where $E_{f}$ is the enthalpy of the fluid, $T_{f}$ the temperature field, and $\mathrm{k}_{\text {eff }}$ the conductivity coefficient of the fluid. equipped with a long distance microscope , recorded frames can reach 298, 851 frames per second (fps). The frames are illuminated with a cold light resource (150 Watts), which is laid opposite beside of the chamber separately.

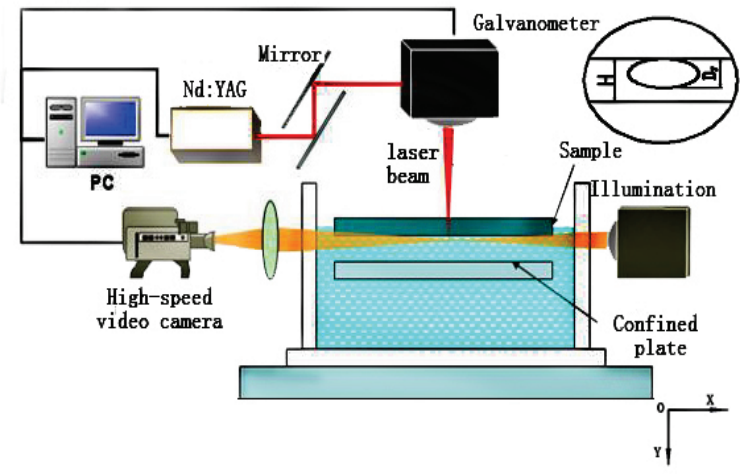

Fig. 1 Experiment setup

\section{Theoretical model}

The VOF method based on a piecewise linear interface calculation (PLIC) method has been adopted. The model is based on Youngs' VOF method which the interface is approximated by a straight-line segment in each cell, but the line could be oriented arbitrarily with respect to the coordinate axis. The orientation of the line is determined by the unit normal vector to the interface and the average value of the cell volume fraction $F$ in the cell under consideration, as well as neighboring cells, and the unit normal vector is calculated using the volume fraction field, therefore this method is only based on volume fraction data, accounting for substantial topology changes in interface. As a result, Youngs' VOF method gives the best overall performance in standard two-dimensional (2D) advection tests and simulations of 2D Rayleigh-Taylor equation.

\subsection{Governing equations}

The flow is assumed to be laminar, the liquid phase is incompressible, and the gas phase is assumed to be an ideal gas without considering mass transfer ${ }^{[14]}$. The VOF method is used to track the interface between the liquid and the gas phases. The governing conservation equations for unsteady, incompressible, Newtonian, multi-fluid flows are given by the following expressions:

The continuity equation is:

$$
\frac{\partial \rho}{\partial \mathrm{t}}+\nabla \cdot(\rho \vec{v})=0
$$

where $\rho$ is the fluid density and $\vec{v}$ the velocity field in the whole domain.

The energy equation is:

$$
\frac{\partial}{\partial \mathrm{t}}\left(\rho E_{f}+\nabla \cdot\left(\vec{v}\left(\rho E_{f}+P_{f}\right)\right)=\nabla \cdot\left(k_{e f f} \nabla T_{f}\right)\right.
$$

The momentum equation is: 


$$
\frac{\partial}{\partial \mathrm{t}}(\rho \vec{v})+\nabla \cdot(\rho \vec{v} \vec{v})=-\nabla P_{f}+\nabla \cdot\left[\mu\left(\nabla \vec{v}+\nabla \vec{v}^{T}\right)\right]+\rho \vec{g}+\vec{F}_{\mathrm{s}}
$$

Where $\mathrm{P}_{\mathrm{f}}$ is the pressure domain, $\mathrm{g}$ is the gravity force. $\mathrm{F}_{\mathrm{s}}$ is the surface tension force with formula of $F_{S}=\sigma k \vec{m}$, where $\kappa$ is the curvature and $\vec{m}$ the normal vector to the interface.

As the VOF method is based on a one dimensional fluid approach, the fluid density and viscosity are calculated according to the following equations:

$$
\begin{aligned}
& \rho=\rho_{1} F+\rho_{g}(1-F) \\
& \mu=\mu_{l} F+\mu_{g}(1-F)
\end{aligned}
$$

Here $\mathrm{F}$ is the equation of volume fraction, the volume fraction $\mathrm{F}$ evolves through the solution of the following equation:

$$
\frac{D F}{D \mathrm{t}}=\frac{\partial F}{\partial \mathrm{t}}+(\vec{v} \cdot \nabla F)=0
$$

\subsection{Calculation model}

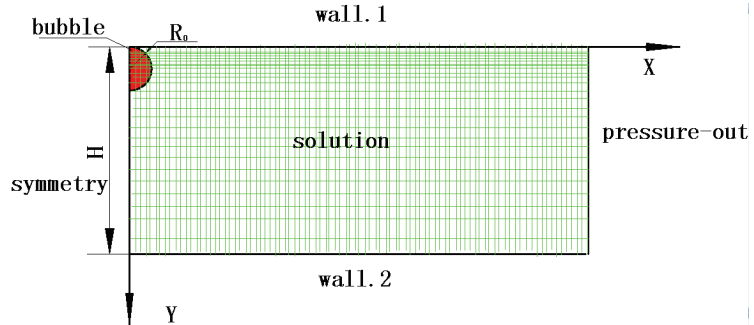

Fig. 2 Schematic diagram of the solution domain, boundary conditions and grid generation

Because the bubble motion is axisymmetric and the computing results can be displayed in the form of mirror symmetry, only $1 / 2$ area is computed to simplify the calculation quantity and reduce the calculation time.

A schematic of the solution domain is showed in Fig. 2. The size of the calculation area in the $X$ axis direction is $2.5 \mathrm{~mm}$ and the gap height $\mathrm{H}$ along $\mathrm{Y}$ axis is of $0.43,1.25,2.5,3.75$ and $10 \mathrm{~mm}$, respectively. The red semicircle region of the area is the laser induced initial bubble with a radius of $R_{0}$. Boundary conditions are defined as follows: The substrate above the bubble is considered as wall.1, the confined plate is regarded as wall.2, the left edge is axis of symmetry, and the right edge connected to the atmosphere is pressure-out.

A quadratic structured, 2D grid is generated to simulate the bubble dynamic and flow field. The grid along $\mathrm{X}$ axis is divided evenly, but the grid along $\mathrm{Y}$ axis increase with a certain ratio greater than 1. As a result, the grid number in the region around the initial bubble is larger than other regions. It is conducive to shorten the computing time under the precondition of good grid quality in this way.
The finite volume method via the pressure based segregated algorithm is employed to discretize the unsteady and laminar equations (Eqs.1-5). The PRESTO scheme is employed to discretize the pressure equation, and a first order upwind scheme is adopted to discretize the convective and diffusive fluxes in momentum and energy equations. The pressure implicit with splitting of operators (PISO) algorithm on a collocated grid is adopted for pressure-velocity coupling.

\subsection{The initial value of the simulation}

The initial pressure, temperature and specific heat capacity of the gas inside the bubble are determined in the following way.

The initial specific internal energy $e$ inside the bubble can be calculated as follows ${ }^{[15]}$ :

$$
e=e_{0}+\Delta e
$$

Here $e_{0}$ is the initial specific internal energy of the water, $\Delta e$ the increased specific internal energy of the gas after absorbing the laser energy $E_{a}$, which is proportional to the single pulse laser energy ${ }^{[16]}$, the increased specific internal energy is defined as follows ${ }^{[15]}$ :

$$
\Delta e=\frac{E_{a}}{M}=\frac{E_{a}}{\frac{4}{3} \pi R_{0}^{3} \rho_{0}}
$$

Here $\rho_{0}$ is the initial density of gas. $\mathrm{R}_{0}$ is the initial radius of the bubble.

The ideal gas equations are expressed as follows:

$$
e=\frac{i}{2} v p=\frac{i}{2} R T
$$

Here $v$ is the specific volume of the gas, $i$ the gas degrees of freedom. In terms of monatomic molecule, $\mathrm{i}$ is of 3 , diatomic molecules $\mathrm{i}$ of 5 , polyatomic molecule $\mathrm{i}$ of 6 . $\mathrm{R}$ is the commonly used constant of gas, $\mathrm{T}$ is the initial temperature.

Combine Eqs. (7), Eqs. (8) with Eqs. (9), we can then get formulas about $\mathrm{P}$ and $\mathrm{T}$ as follows:

$$
\begin{aligned}
& p=\frac{2 e_{0}}{i v}+\frac{E_{a}}{\frac{4}{3} \pi R_{0}^{3} \rho_{0} i v} \\
& T=\frac{2 e_{0}}{i R}+\frac{E_{a}}{\frac{4}{3} \pi R_{0}^{3} \rho_{0} i R}
\end{aligned}
$$

For this cases, $\mathrm{e}_{0}=104.83 \mathrm{~kJ} / \mathrm{kg}, \rho_{0}=53.87$ $\mathrm{kg} / \mathrm{m}^{3}, \quad v=0.0186 \mathrm{~m}^{3} / \mathrm{kg}, \quad \mathrm{R}=461.5 \mathrm{~J} / \mathrm{Kg} \cdot \mathrm{K}^{-1}$. By setting $\mathrm{R}_{0}=0.02 \mathrm{~mm}, E_{a}=0.04 \mathrm{~mJ}$, the vapor degrees of freedom $i=6$, the initial pressure inside a bubble is finally calculated as $45 \mathrm{MPa}$, the initial temperature is $1810 \mathrm{~K}$.

And, furthermore, the density of liquid phase is recognized as $1250 \mathrm{~kg} / \mathrm{m}^{3}$, because the used liquid 
is $\mathrm{CuSO}_{4}$ solution with a concentration of $20 \%$. Some key initial parameters of the simulation are listed as follows:

Table 1 The initial parameters of the simulation

\begin{tabular}{cccccc}
\hline & $\mathrm{P} / \mathrm{Pa}$ & $\mathrm{T} / \mathrm{K}$ & $\delta / \mathrm{w} /(\mathrm{m} \cdot \mathrm{K})^{-1}$ & $\eta / \mathrm{kg} \bullet(\mathrm{m} \cdot \mathrm{s})^{-1}$ & $\mathrm{Cp} / \mathrm{J} \cdot(\mathrm{kg} \cdot \mathrm{K})^{-1}$ \\
\hline Gas & 45000000 & 1810 & 0.0261 & $1.34 \mathrm{e}-5$ & 2668.5 \\
Liquid & 101325 & 300 & 0.6 & 0.001 & 3800 \\
\hline
\end{tabular}

\section{Results and discussion}

We succeed in obtaining the continuous images of asymmetric bubbles which are created at the varied height gaps. The bubble behavior and the flow field distribution are reported below. When the liquid gap height is higher than $1.25 \mathrm{~mm}$, the bubble behavior and the flow field distribution show great similarity with certain regularity, therefore in the following section, the article only introduce the general rules of bubble dynamic and elaborate $10 \mathrm{~mm}$, $1.25 \mathrm{~mm}, 0.43 \mathrm{~mm}$ in detail. It should be pointed out that in all cases, the upper boundary is sapphire sample and the bottom boundary is the confined plate, but the confined plate is marked out only in the case for $\mathrm{H}=0.43 \mathrm{~mm}$.

\subsection{Effects on bubble diameter by variable gap height}

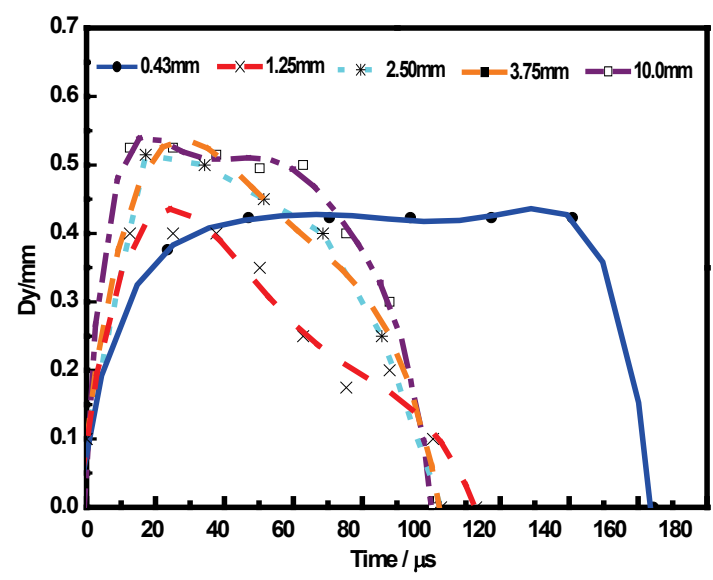

Fig. 3 The diameter Dy along y direction of bubble dynamics at variable gap height $\mathrm{H}$

As we know, different values of $\mathrm{H}$ mainly mean different degree of restriction in the laser incident direction (y direction). Therefore, it is of great importance to study the change law of the cavitation bubble diameter along $y$ direction $\left(D_{y}\right)$ at varied height gaps. Fig. 3 shows the bubble diameter varies as a function of $\mathrm{H}$, whose data is obtained from the experiment. The general rule is that with the decreasing $\mathrm{H}$, the lifetime of the bubble increases and the maximum value of $\mathrm{D}_{\mathrm{y}}$ decreases. The thinnest gap, $\mathrm{H}=0.43$ $\mathrm{mm}$, the lifetime of the bubble is about $170 \mu \mathrm{s}$, in other cases, bubble pulsation cycle is around $110 \mu \mathrm{s}$, but when $\mathrm{H}=1.25 \mathrm{~mm}$, the lifetime is slightly larger due to its second pulsation. Interestingly, when $\mathrm{H}=0.43 \mathrm{~mm}$, the $\mathrm{D}_{\mathrm{y}}$ increases to maximum and remains the same about $100 \mu \mathrm{s}$. Then the bubble reduces quickly during the last stage of collapse. This is due to the great limitation by the confined plate, leading to the restriction of the bubble growth in the $y$ direction. The smaller the gap, the higher inhibition for bubble motion, therefore the bubble lifetime is longer. Because the pressure and temperature in the bubble is high, it may be beneficial to decompose the $\mathrm{CuSO}_{4}$ component in solution and generate photochemical composition on the rear sapphire surface.

\subsection{The case for $H=10 \mathrm{~mm}$}

The bubble motion and the flow field for $\mathrm{H}=10 \mathrm{~mm}$ are shown in Figure 4. Fig. 4 (a) shows a typical kind of bubble shape change form, taken at a framing rate of 79649 fps. The first picture of the continuous images shows that a bright plasma flashing appeals at the moment of bubble generated. Afterwards, the bubble grows semi-spherically till reaching the maximum volume. The dented bubble in pictures (frames 5-10) indicate that the liquid jet is formed at the top of the bubble when it starts collapsing, hits the far bubble wall in the final stage of the collapse and penetrates the bubble.

Fig. 4 (b) \& (c) are the simulation results, which are assisting in interpreting the bubble dynamics. Fig. 4 (b) is the contours of the vapor volume fraction, the calculated bubble lifetime is $116 \mu \mathrm{s}$, and the maximal radius of bubble is about $0.52 \mathrm{~mm}$, which agrees well with the experimental results.

Fig. 4(c) are the several representative flow field distribution pictures in the case of $\mathrm{H}=10 \mathrm{~mm}$. During the expansion process, the fluid presents a radial distribution developing from bubble center (picture $\mathrm{t}=4.6 \mu \mathrm{s}$ ), the flow field is asymmetric distribution with the main flow pointing to the below, due to the limitation of the upper sample. The maximum expansion speed value is around 50 $\mathrm{m} / \mathrm{s}$. When the bubble expands to the maximum, the flow evolves into two vortex on the left and right side of the bubble close to the sapphire (picture $\mathrm{t}=58.5 \mu \mathrm{s}$ ). At this time, the velocity is very small, only $1 \mathrm{~m} / \mathrm{s}$. During the bubble collapse process, the flow jet produces at the top of the bubble and points to the sample surface with a velocity of $15.4 \mathrm{~m} / \mathrm{s}$ (picture $\mathrm{t}=110.5 \mu \mathrm{s}$ ). Then the jet develops into two divided jets (picture $\mathrm{t}=118.5 \mu \mathrm{s}$ ), which are the result of the jet impacting on the wall. The two divided jets depart from the processing surface with a small acute angle. Fig. 4(d) shows the macroscopic fluid distribution in the process of laser processing, which can be regarded as the cumulative effects of multiple laser pulses. There are three main streams, the left and right streams originate from the two divided jets in the picture when $\mathrm{t}=118.5 \mu \mathrm{s}$, and the 
downward stream stems from the flow formed in the expansion process.

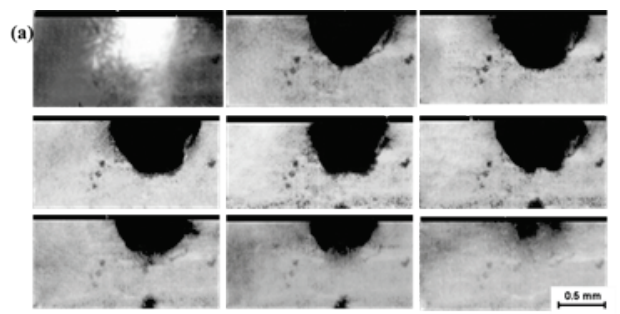

(b)

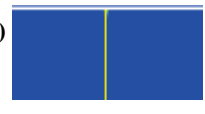

0us

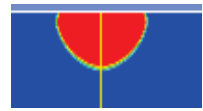

$45 \mu \mathrm{s}$

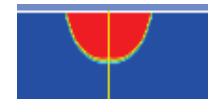

$90 \mu \mathrm{s}$

(c)

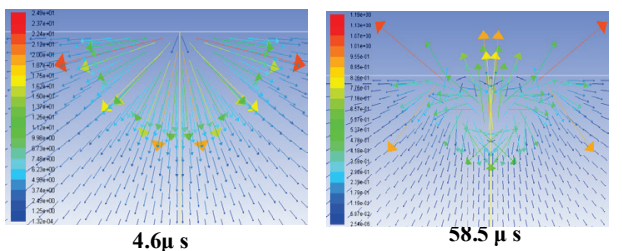

$4.6 \mu \mathrm{s}$

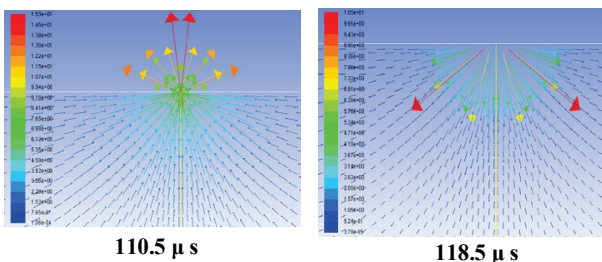

(d)

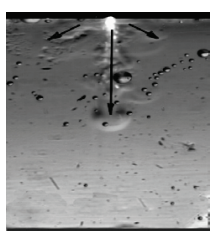

Fig. 4 (a) High speed photos of laser induced cavitation bubble at a gap height of $10 \mathrm{~mm}$. Liquid concentration of $20 \%$, frequency of $2 \mathrm{kHz}$, single pulse energy of $4.0 \mathrm{~mJ} /$ pluses, inter-frame time of $12.56 \mu \mathrm{s}$ and exposure time of $8 \mu \mathrm{s}$; (b) Contours of the gas volume fraction by simulation; and (c) Flow field distribution for $\mathrm{H}=10 \mathrm{~mm}(\mathrm{~d})$ the macroscopic fluid distribution for $\mathrm{H}=10 \mathrm{~mm}$

\subsection{The case for $H=1.25 \mathrm{~mm}$}

Figure 5 illustrates the whole process of bubble motion and the flow field for $\mathrm{H}=1.25 \mathrm{~mm}$. Fig. 5 (a) is the photographic sequences with inter-frame time of $12.56 \mu \mathrm{s}$. In this situation, the induced bubble experiences three dynamical stages, including bubble expansion, bubble collapse, and bubble re-expansion.

The experiment shows that the bubble grows in the same way as the case for $\mathrm{H}=10 \mathrm{~mm}$. The differences are that the bubble collapses semi-spherically and has a reexpansion course. Fig. 5 (a) (8-10) shows the bubble rebounds with a maximal radius of $0.1 \mathrm{~mm}$, then contracts again and disappears in the end. The whole re-expansion process lasts for about $30 \mu \mathrm{s}$, accounting $25 \%$ of the total time. However, the simulation result does not appear bubble re-expansion. The reason why the bubble rebounds is that the bubble collapses so quickly that the energy in the bubble cannot be dissipated.

The flow field around the bubble is similar to the case for $\mathrm{H}=10 \mathrm{~mm}$, only the maximum speed of flow expansion and collapse speed of flow is $138 \mathrm{~m} / \mathrm{s}$ and $22.3 \mathrm{~m} / \mathrm{s}$, respectively.

(a)

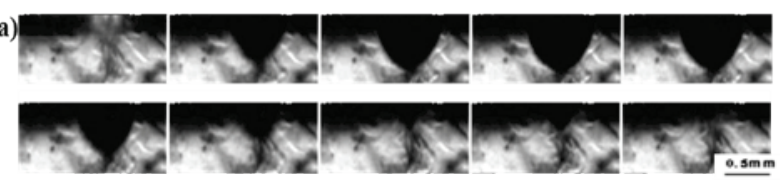

(b)

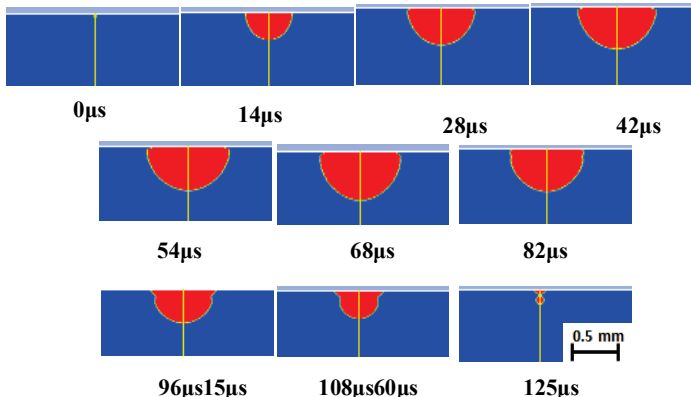

Fig. 5 (a)High speed photos of laser induced cavitation bubble at a gap height of $1.25 \mathrm{~mm}$. Liquid concentration of $20 \%$, frequency of $2 \mathrm{kHz}$, single pulse energy of $4.0 \mathrm{~mJ} /$ pluses, interframe time of $12.56 \mu$ s and exposure time of $8 \mu \mathrm{s}$; and (b) Contours of the vapor volume fraction by simulation

\subsection{The case for $\mathrm{H}=\mathbf{0 . 4 3} \mathrm{mm}$}

Figure 6 shows the dynamics of a bubble created at the gap height of $0.43 \mathrm{~mm}$. Fig. 6 (a) illustrates the high-speed sequence recorded at $40316 \mathrm{fps}$. The bubble behavior is different from all the other cases and is explained as follows. After laser irradiation, the bubble expands quickly and semi-elliptically. At $\mathrm{t}=49.6 \mu \mathrm{s}$, the bubble's top boundary approaches the confined plate, and then the bubble expands toward the horizontal direction, due to the strong restrictions of the confined plate. The bubble reaches the maximum volume at $\mathrm{t}=74.4 \mu \mathrm{s}$. Subsequently, the bubble begins collapsing along the horizontal direction and disappears in the last. Fig. 6 (b) is the simulation result of the gas volume fraction contours, which is consistent with the experimental results well. At this case, the flow field distribution is roughly as same as $\mathrm{H}=10 \mathrm{~mm}$, but also has a difference, in the process of the original expansion stage, there is very high speed flow pointing to the sample wall shown in the figure when $\mathrm{t}=0.1 \mu \mathrm{s}$, and the liquid distribute above the bubble is squeezed more than other cases (picture $\mathrm{t}=61 \mu \mathrm{s}$ ), In the process of collapse, the left and right jets are formed toward the bubble center (picture $\mathrm{t}=143.5 \mu \mathrm{s}$ ), obliquely striking on the substrate surface at $\mathrm{t}=138 \mu \mathrm{s}$, the maximum speed is $32 \mathrm{~m} / \mathrm{s}$. 
(a)
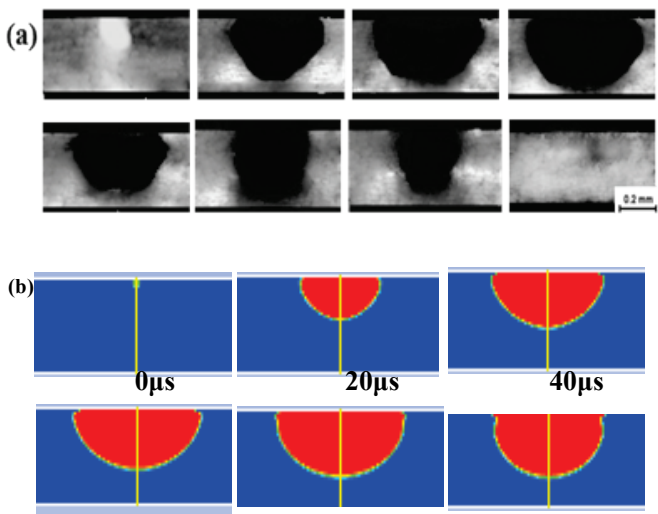

$60 \mu \mathrm{s}$

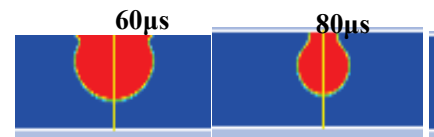

$120 \mu s$

$140 \mu s$

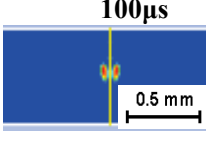

$168 \mu \mathrm{s}$

(c)

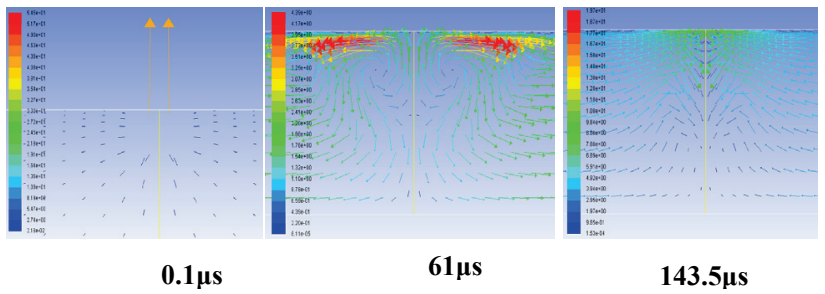

Fig. 6 (a) High speed photos of laser induced cavitation bubble at a gap height of $0.43 \mathrm{~mm}$. Liquid concentration of $20 \%$,frequency of $2 \mathrm{kHz}$, single pulse energy of 4.0 $\mathrm{mJ} /$ pluses, interframe time of $24.8 \mu \mathrm{s}$ and exposure time of $8 \mu \mathrm{s}$;

(b) Contours of the vapor volume fraction by simulation; and (c) Flow field distribution for $\mathrm{H}=0.43 \mathrm{~mm}$

In the process of expansion, the fluid distribution can make the liquid to have a good contact with the machining surface and accelerate the composition in the liquid deposit on the rear sapphire surface, the sediments can strengthen the absorption of laser energy, and then the material is melted quickly. Because the jets produced in the process of collapse do not vertically impact on the machining sample, it is good for the flow to bring out the melted sapphire mainly formed in the bubble expansion course and the debris deposited at the bottom of the processing groove. Therefore, the fluid distribution is conductive to increase the etching rate and improve the sample surface quality.

Furthermore, from the macroscopic flow field distribution showed in Fig.4 (d), we can deduce that the three streams will have a collision with the plate and produce a backflow. If the below confined plate is very close to the substrate, it will speed up the convection, update the composition in the liquid and have a great influence on the next pulse function, which is also contribute to the composition in the liquid deposit on the surface and increased the etching rate.

\subsection{Experiment validation}

Based on the above study of the bubble dynamic and our forecast, validation testes are carried out. The used liquid is $\mathrm{CuSO}_{4}$ solution with the concentration of $20 \%$. The pulse laser energy is $4.0 \mathrm{~mJ}$, the frequency of laser is 2 $\mathrm{kHz}$, the scanning speed is $5 \mathrm{~mm} / \mathrm{s}$ and the scanning times ranges from 4 to 8 . A laser confocal microscope (LEXT

OLS4000, Olympus) is used to measure the processed groove size characteristics.

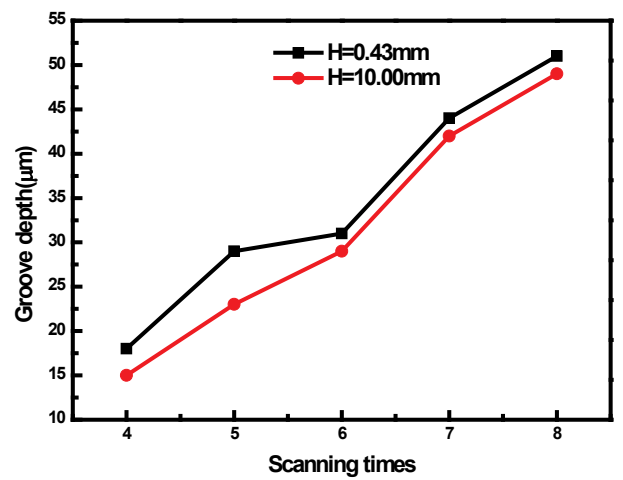

Fig.7 Groove depth as a function of scan times. Liquid concentration of $20 \%$, frequency of $2 \mathrm{kHz}$ and scanning speed of $5 \mathrm{~mm} / \mathrm{s}$

(a)

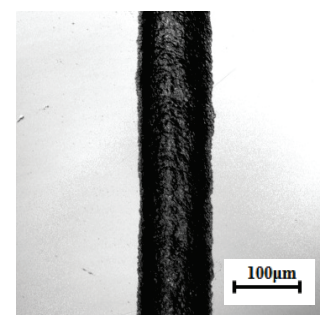

(c)

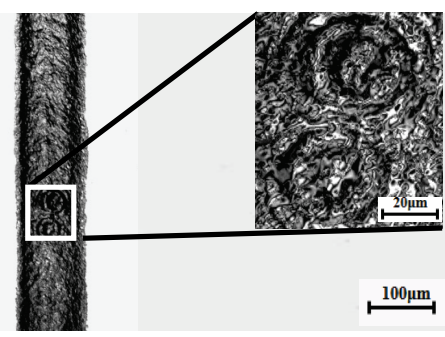

Fig. 8 (a)The processed surface quality for $\mathrm{H}=0.43 \mathrm{~mm}$;(b) The three-dimensional view of the surface quality for $\mathrm{H}=0.43 \mathrm{~mm}$; and (c) The processed surface quality for $\mathrm{H}=10 \mathrm{~mm}$. Liquid concentration of $20 \%$, scanning speed of $2 \mathrm{~mm} / \mathrm{s}$, scanning times of 2 ; single pulse energy of $4.0 \mathrm{~mJ} /$ pluses

Figure 7 shows that the groove depth increases as a function of scan times. The etching rate in the case for $\mathrm{H}=$ $0.43 \mathrm{~mm}$ is higher than the case for $\mathrm{H}=10 \mathrm{~mm}$ at the same laser etching parameters. This is mainly owing to the longer lifetime of bubble and the liquid distribution, which is useful to generate photochemical deposition on the rear sapphire surface. Fig. 8(a) and (b) show optical microscope images of front and 3D views of sapphire substrates by using laser-induced back side wet etching method at the gap height of $0.43 \mathrm{~mm}$, respectively. Fig. $8(\mathrm{c})$ is the processed surface quality for $\mathrm{H}=10 \mathrm{~mm}$. In this case, the used liquid concentration is $20 \%$ and scanning speed 2 $\mathrm{mm} / \mathrm{s}$, scanning times 2. Fig. 8(a) and (b) demonstrate that the sample gained a good quality surface with a small heat affected zone and a neat smooth bottom. However, the processed surface of $\mathrm{H}=10 \mathrm{~mm}$ easily appears crater morphology (Fig. 8(c)), because the liquid convection is not faster than that at the smaller gap height, the liquid around the processed zone is easier to produce irregular boiling bubbles, when these bubbles collapse on the melted 
area, the crater is produced. Figure 7 and 8 commonly prove that the cavitation bubbles at a certain gap height can help to increase the etching rate and improve the quality of the machining surface.

\section{Conclusions}

In this paper, we study the influence on the laserinduced cavitation bubble dynamics by changing the gap height through experiments and numerical simulation, and explore the mechanism of the enhanced etching rate and proved quality processed by $1064 \mathrm{~nm}$ pulsed laser in $\mathrm{CuSO}_{4}$ solution. Several conclusions are drawn as followers:

(1) With the decreasing $\mathrm{H}$, the lifetime of the bubble increases and the maximum value of $\mathrm{D}_{\mathrm{y}}$ decreases. When $\mathrm{H}=0.43 \mathrm{~mm}$, the lifetime of the bubble is the longest, in other cases, bubble pulsation cycles close to the same value, but when $\mathrm{H}=1.25 \mathrm{~mm}$, the lifetime is slightly larger due to its second pulsation. Interestingly, when $\mathrm{H}=0.43 \mathrm{~mm}$, the $D_{y}$ increases to maximum and remains the same about $100 \mu$ s. Then the bubble reduces quickly.

(2) When the liquid gap height is higher than $1.25 \mathrm{~mm}$, the fluid presents a radial distribution developing from bubble center during the expansion process. During the bubble collapse process, the flow jet points to the sample surface. When $\mathrm{H}=0.43 \mathrm{~mm}$, there is very high speed flow pointing to the sample wall and the liquid distribute above the bubble is squeezed more than other cases In the process of collapse, jets are striking on the substrate surface obliquely.

(3) Validation testes proves that the etching rate in the case for $\mathrm{H}=0.43 \mathrm{~mm}$ is higher than the case for $\mathrm{H}=10 \mathrm{~mm}$ at the same laser etching parameters. This is mainly owing to the longer lifetime of bubble and the liquid distribution. Besides, the faster liquid convection, the better heat dissipation, this is good to improve the processed surface quality.

\section{Acknowledgments}

The research is supported by National Nature Science Foundation of China (No.50805027, 50675038), Guangdong Natural Science Foundation (No.S2013010014070) and Open Fund of Jiangsu Key Laboratory of Precision and Micro-manufacturing Technology.

\section{References}

[1] X.Z. Xie, Z.Y. Pan, X. Wei, W. Hu and M.H. Hong: Journal Of Laser Micro Nanoengineering, 6, (2011), 209.

[2] Z.Q. Huang, M.H. Hong, T.B.M. Do and Q.Y. Lin: Applied Physics A: Materials Science \& amp. 93, (2008), 159.

[3] P. Schwallera, S. Zehnder, U. von Arxb, B. Neuenschwander: Physics Procedia., 12,(2011)188.

[4] Ximing Ding, Tadatake Sato, Yoshizo Kawaguchi and Hiroyuki Niino: Japanese Journal of Applied Physics, 42, (2003) 176.

[5] VAKochemirovsky, LGMenchikov and SVSafonov: Russian Chemical Reviews, 80, (2011) 869.

[6] R. Bohme and K. Zimmer: Applied Surface Science, 247, (2005) 256.
[7] S. R. Gonzalez-Avila, E. Klaseboer B. C. Khoo and C.D. Ohl: Journal of Fluid Mechanics, 682, (2011)241.

[8] I.Akhatov, O.Lindau, A.Topolnikov, R.Mettin, N. Vakhitova, N. Vakhitova, W. Lauterborn and W.Lauterborn: 13, (2001) 2805.

[9] Y.L. Zhang, K. Yeo, B.C. Khoo and C. Wang: Journal of Computational Physics, 166, (2001) 336.

[10]J.T. Huang and H.S. Zhang: Springer-Verlag, 23, (2007)645.

[11] Xu Ling-jun, Chen Gang, Shao Jian-bin and Xue Yang: Journal of 2011 International Conference on Electric Technology and Civil Engineering, 13, (2011)2408.

[12] M. Cheng, J.S. Hua and J. Lou: Computers \& Fluids, $39,(2010) 260$.

[13] Mark Sussman: Journal of Computational Physics, 187, (2003)110.

[14] M. van Sint Annaland, N.G. Deen and J.A.M. Kuipers: Chemical Engineering Science, 60, (2005)2999.

[15] Yuhong Wang, Jiangan Wang and Ronghua Wu: High Power Laser and Particle Beams, 21, (2009) 998.

[16]X. W Ni, X. Chen, J. Lu: Laser Technology, 26, (2002)258

(Received: August 7, 2013, Accepted: November 29, 2013) 